\title{
Gout and Hypothyroidism in the Elderly: an Observational Cohort Study Using U.S. Medicare Claims Data
}

\author{
Jasvinder A. Singh, MBBS, MPH ${ }^{1,2}$ and John D. Cleveland, $\mathrm{MS}^{2}$ \\ ${ }^{1}$ Medicine Service, VA Medical Center, Birmingham, AL, USA; ${ }^{2}$ Department of Medicine at School of Medicine, Division of Epidemiology at School \\ of Public Health, University of Alabama at Birmingham, Birmingham, AL, USA.
}

J Gen Intern Med 33(8):1229-31

DOI: $10.1007 / \mathrm{s} 11606-018-4460-4$

(c) Society of General Internal Medicine (This is a U.S. Government work and not under copyright protection in the US; foreign copyright protection may apply) 2018

\section{INTRODUCTION}

Given the known association of hypothyroidism and hyperuricemia, a link between gout and hypothyroidism has long been suspected, but rarely investigated. Two small studies in hospitalized patients ${ }^{1}$ or at a single medical center ${ }^{2}$ suggested a higher risk of hypothyroidism in people with gout. These studies included small samples, controlled for only select demographic variables and none focused on the elderly, the population at the highest risk of hypothyroidism. Welldesigned studies that can control for potential confounders can provide a robust estimate of this potential association are needed. Our objective was to examine whether gout, the most common inflammatory arthritis in adults, was associated with an increase in the risk of incident hypothyroidism in adults 65 years or older.

\section{METHODS}

We used the 5\% random Medicare claims data to conduct this cohort study. Patients were considered eligible for this analysis, if they received Medicare fee-for-service (parts A or B) coverage and were not enrolled in a Medicare Advantage Plan (part C) during 2006-12 and had a valid US address. Medicare denominator file and claims data including prescription data (part D) provided predictor, outcome, and covariate data. The Institutional Review Board at the University of Alabama at Birmingham (UAB) approved the study.

The predictor of interest was gout, identified by the presence of two ICD-9-CM diagnostic codes, 274.xx, in the claims data, a valid approach with high accuracy with sensitivity of $90 \%$ and specificity of $100 \% .^{3}$ In all patients with gout and hypothyroidism during the study period, the diagnosis of gout had to precede the diagnosis of hypothyroidism. The study outcome was incident hypothyroidism, identified by the new occurrence (baseline period, 365 days) of at least two ICD-9$\mathrm{CM}$ codes at least 4 weeks apart using the following ICD-9CM codes, ${ }^{4}$ 243.xx, 244.xx, 240.9, 246.1, and 246.8, a valid

Published online April 30, 2018 approach. ${ }^{5}$ People were censored at the time of diagnosis of hypothyroidism, death, or the end of the study period, 12/31/ 2012, whichever occurred first.

We used a multivariable-adjusted Cox proportional hazard to assess the independent association of gout with incident hypothyroidism, adjusting for important covariates and confounders including patient demographics (age, race, sex), common medications for cardiovascular disease (statins, beta-blockers, diuretics, and angiotensin-converting enzyme (ACE)-inhibitors) and urate-lowering medications for gout (allopurinol and febuxostat), and medical comorbidity assessed using the Charlson-Romano index, a commonly used validated weighted comorbidity index consisting of $17 \mathrm{com}$ mon comorbidities, calculated using the claims data (model 1). Sensitivity models categorized Charlson-Romano index (model 2) or adjusted for all 17 Charlson-Romano comorbidities (plus hypertension, hyperlipidemia and coronary artery disease; model 3). We did not include outpatient utilization in the model due to collinearity with comorbidity, as previously demonstrated.

\section{RESULTS}

We found that 168,258 of the 1.57 million Medicare recipients, 8950 with gout and 159,308 without gout, developed incident (new diagnosis of) hypothyroidism during the followup. Mean/median time from a diagnosis of gout to new diagnosis of hypothyroidism was 2.2/1.9 years (standard deviation, 1.6; inter-quartile range, $0.8,3.3$ ). Compared to people who did not develop hypothyroidism, people who developed hypothyroidism were more likely to female, White, and have higher medical comorbidity (Table 1). Crude incidence rates of hypothyroidism per 1000 person-years were 38.0 in people with gout and 18.5 in people without gout.

Gout was associated with 1.8-times hazards of incident hypothyroidism in the elderly in multivariable-adjusted model, confirmed in sensitivity analyses that substituted continuous Charlson-Romano index score with categorized score or individual comorbidities (Table 2). Older age, female gender, White race, and higher medical comorbidity score were also associated with higher hazard ratios of incident hypothyroidism (Table 2). 
Table 1 Demographic and clinical characteristics of episodes of incident hypothyroidism

\begin{tabular}{|c|c|c|c|c|}
\hline & \multirow[t]{2}{*}{ Entire cohort } & \multicolumn{2}{|c|}{ Hypothyroidism during the follow-up } & \multirow[t]{2}{*}{$P$ value } \\
\hline & & No & Yes & \\
\hline Total, $N$ & $1,572,874 *$ & $1,404,616$ & 168,258 & \\
\hline Age, mean (SD) & $75.2(7.6)$ & $75.2(7.6)$ & $75.1(7.2)$ & $<0.0001$ \\
\hline Gender, $N(\%)$ & & & & $<0.0001$ \\
\hline Male & $704,184(44.8 \%)$ & $648,664(46.2 \%)$ & $55,520(33.0 \%)$ & \\
\hline Female & $868,690(55.2 \%)$ & $755,952(53.8 \%)$ & $112,738(67.0 \%)$ & \\
\hline Race/ethnicity, $N(\%)$ & & & & $<0.0001$ \\
\hline White & $1,345,283(85.5 \%)$ & $1,197,683(85.3 \%)$ & $147,600(87.7 \%)$ & \\
\hline Black & $135,664(8.6 \%)$ & $124,914(8.9 \%)$ & $10,750(6.4 \%)$ & \\
\hline Other/unknown & $91,927(5.8 \%)$ & $82,019(5.8 \%)$ & $9908(5.9 \%)$ & \\
\hline Charlson-Romano comorbidity score, mean (SD) & $1.55(2.38)$ & $1.53(2.39)$ & $1.71(2.29)$ & $<0.0001$ \\
\hline Charlson-Romano score & & & & $<0.0001$ \\
\hline 0 & $852,801(54.2 \%)$ & $775,180(55.2 \%)$ & $77,621(46.1 \%)$ & \\
\hline 1 & $153,480(9.8 \%)$ & $132,884(9.5 \%)$ & $20,596(12.2 \%)$ & \\
\hline$\geq 2$ & $566,593(36.0 \%)$ & $496,552(35.4 \%)$ & $70,041(41.6 \%)$ & \\
\hline \multicolumn{5}{|l|}{ Charlson-Romano comorbidities } \\
\hline Myocardial infarction & $61,332(3.9 \%)$ & $54,372(3.9 \%)$ & $6960(4.1 \%)$ & $<0.0001$ \\
\hline Heart failure & $177,478(11.3 \%)$ & $156,066(11.1 \%)$ & $21,412(12.7 \%)$ & $<0.0001$ \\
\hline Peripheral vascular disease & $148,510(9.4 \%)$ & $130,043(9.3 \%)$ & $18,467(11.0 \%)$ & $<0.0001$ \\
\hline Cerebrovascular disease & $147,378(9.4 \%)$ & $129,112(9.2 \%)$ & $18,266(10.9 \%)$ & $<0.0001$ \\
\hline Dementia & $69,248(4.4 \%)$ & $63,130(4.5 \%)$ & $6118(3.6 \%)$ & $<0.0001$ \\
\hline Chronic pulmonary disease & $236,532(15.0 \%)$ & $207,780(14.8 \%)$ & $28,752(17.1 \%)$ & $<0.0001$ \\
\hline Connective tissue disease & $40,109(2.6 \%)$ & $34,133(2.4 \%)$ & $5976(3.6 \%)$ & $<0.0001$ \\
\hline Peptic ulcer disease & $28,573(1.8 \%)$ & $24,843(1.8 \%)$ & $3730(2.2 \%)$ & $<0.0001$ \\
\hline Mild liver disease & $7366(0.47 \%)$ & $6432(0.46 \%)$ & $934(0.56 \%)$ & $<0.0001$ \\
\hline Diabetes & $281,214(17.9 \%)$ & $244,405(17.4 \%)$ & $36,809(21.9 \%)$ & $<0.0001$ \\
\hline Diabetes with end organ damage & $82,380(5.2 \%)$ & $71,331(5.1 \%)$ & $11,049(6.6 \%)$ & $<0.0001$ \\
\hline Hemiplegia & $12,669(0.81 \%)$ & $11,392(0.81 \%)$ & $1277(0.76 \%)$ & 0.024 \\
\hline Renal failure/disease & $52,100(3.3 \%)$ & $46,049(3.3 \%)$ & $6051(3.6 \%)$ & $<0.0001$ \\
\hline Any tumor, leukemia, or lymphoma & $154,604(9.8 \%)$ & $136,403(9.7 \%)$ & $18,201(10.8 \%)$ & $<0.0001$ \\
\hline Moderate or severe liver disease & $1782(0.11 \%)$ & $1603(0.11 \%)$ & $179(0.11 \%)$ & 0.37 \\
\hline Metastatic cancer & $16,361(1.0 \%)$ & $15,043(1.1 \%)$ & $1318(0.78 \%)$ & $<0.0001$ \\
\hline AIDS & $521(0.03 \%)$ & $459(0.03 \%)$ & $62(0.04 \%)$ & 0.37 \\
\hline Hypertension & $724,828(46.1 \%)$ & $630,361(44.9 \%)$ & $94,467(56.1 \%)$ & $<0.0001$ \\
\hline Hyperlipidemia & $515,307(32.8 \%)$ & $444,529(31.6 \%)$ & $70,778(42.1 \%)$ & $<0.0001$ \\
\hline Coronary artery disease & $266,728(17.0 \%)$ & $231,430(16.5 \%)$ & $35,298(21.0 \%)$ & $<0.0001$ \\
\hline Obesity & $30,881(2.0 \%)$ & $26,601(1.9 \%)$ & $4280(2.5 \%)$ & $<0.0001$ \\
\hline
\end{tabular}

SD standard deviation

*Met eligibility criteria for incident hypothyroidism and did not have hypothyroidism in the baseline 365-day period

Table 2 Association of gout and other risk factors with incident hypothyroidism

\begin{tabular}{|c|c|c|c|c|c|c|}
\hline & \multicolumn{2}{|c|}{$\begin{array}{l}\text { Multivariable-adjusted* } \\
\text { (model 1) }\end{array}$} & \multicolumn{2}{|c|}{$\begin{array}{l}\text { Multivariable-adjusted* } \\
\text { (model 2) }\end{array}$} & \multicolumn{2}{|c|}{$\begin{array}{l}\text { Multivariable-adjusted* } \\
\text { (model 3) }\end{array}$} \\
\hline & HR (95\% CI) & $P$ value & HR $(95 \%$ CI) & $P$ value & HR $(95 \%$ CI) & $P$ value \\
\hline \multicolumn{7}{|l|}{ Age (in years) } \\
\hline $65-<75$ & Ref & & Ref & & Ref & \\
\hline $75-<85$ & $1.18(1.17,1.19)$ & $<0.0001$ & $1.17(1.15,1.18)$ & $<0.0001$ & $1.14(1.13,1.15)$ & $<0.0001$ \\
\hline$\geq 85$ & $1.35(1.33,1.37)$ & $<0.0001$ & $1.34(1.32,1.36)$ & $<0.0001$ & $1.32(1.30,1.34)$ & $<0.0001$ \\
\hline \multicolumn{7}{|l|}{ Gender } \\
\hline Male & Ref & & Ref & & Ref & \\
\hline Female & $1.74(1.72,1.75)$ & $<0.0001$ & $1.74(1.72,1.76)$ & $<0.0001$ & $1.74(1.72,1.76)$ & $<0.0001$ \\
\hline \multicolumn{7}{|l|}{ Race } \\
\hline White & Ref & & Ref & & Ref & \\
\hline Black & $0.66(0.65,0.68)$ & $<0.0001$ & $0.67(0.66,0.69)$ & $<0.0001$ & $0.66(0.65,0.68)$ & $<0.0001$ \\
\hline Other & $0.93(0.91,0.95)$ & $<0.0001$ & $0.95(0.93,0.97)$ & $<0.0001$ & $0.95(0.93,0.97)$ & $<0.0001$ \\
\hline $\begin{array}{l}\text { Charlson-Romano score, } \\
\text { per unit change }\end{array}$ & $1.12(1.12,1.12)$ & $<0.0001$ & N/A & & N/A & \\
\hline \multicolumn{7}{|l|}{ Charlson-Romano score } \\
\hline 0 & $\mathrm{~N} / \mathrm{A}$ & & Ref & & N/A & \\
\hline 1 & & & $1.57(1.55,1.60)$ & $<0.0001$ & & \\
\hline$\geq 2$ & & & $1.80(1.78,1.82)$ & $<0.0001$ & & \\
\hline Gout & $1.76(1.72,1.80)$ & $<0.0001$ & $1.75(1.71,1.79)$ & $<0.0001$ & $1.62(1.58,1.65)$ & $<0.0001$ \\
\hline
\end{tabular}

N/A not applicable, HR hazard ratio, CI confidence interval, Ref referent category

*Model 1 included Charlson-Romano score as a continuous variable; model 2 replaced it with categorized Charlson-Romano score; and model 3 replaced it with each of the 17 Charlson-Romano comorbidities (plus hypertension, hyperlipidemia and coronary artery disease). All models were also adjusted for medications for cardiovascular diseases (statins, beta-blockers, diuretics, ACE-inhibitors) and for urate-lowering therapies for gout (allopurinol, febuxostat). Age and medication use were time-varying covariates

Italics represent statistical significance, with a $P$ value $<0.05$ 


\section{DISCUSSION}

In this study of adult Americans 65 years or older, we found that gout was associated with almost twice the risk of incident hypothyroidism. Gout is associated with hyperuricemia and chronic inflammation, both are linked to hypothyroidism. ${ }^{6}$ Incidence of gout and hypothyroidism increases from 6th decade of life into later years. Elderly with gout may benefit from screening for hypothyroidism. Screening is affordable and early diagnosis and treatment of hypothyroidism may improve patient outcomes.

Study findings must be interpreted considering limitations, such as residual confounding and potential misclassification and ascertainment bias. Study strengths include adjustment for potential confounders, use of a representative large sample, and robustness of findings. Our study advances the knowledge regarding correlates of hypothyroidism and the comorbidity load in gout in the elderly.

\section{Acknowledgements:}

We thank Dr. Jeffrey Curtis of the UAB Division of Rheumatology, who permitted us to re-use the $5 \%$ Medicare data. We thank patients at the University of Alabama gout clinic for asking us questions about comorbidities of gout and whether gout may be related to their other conditions, which prompted us to ask this question.

Funding/Support: This material is the result of work supported by research funds from the Division of Rheumatology at the University of Alabama at Birmingham and the resources and use of facilities at the Birmingham VA Medical Center, Birmingham, Alabama, USA.

Role of the Funder/Supporter: The funding body did not play any role in the design, collection, analysis, and interpretation of data; in the writing of the manuscript; and in the decision to submit the manuscript for publication.

Corresponding Author: Jasvinder A. Singh, MBBS, MPH; Department of Medicine at School of Medicine, Division of Epidemiology at School of Public Health University of Alabama at Birmingham, Birmingham, AL, USA (e-mail: Jasvinder.md@gmail.com).

Author Contributions Mr. Cleveland had full access to all of the data in the study and takes the responsibility for the integrity of the data and accuracy of the data analysis. He was supervised by Dr. Singh, who reviewed all results.

Study Concept and Design: Singh.
Data acquisition, analysis and interpretation of results: Singh, Cleveland

Drafting of the manuscript: Singh

Critical revision of the manuscript for important intellectual content: Singh, Cleveland

Statistical analysis: Cleveland

Obtained Funding: Singh

Administrative, technical or material support: Singh

Study Supervision: Singh

\section{Compliance with Ethical Standards:}

Conflict of Interest: JAS has received research grants from Takeda and Savient and consultant fees from Savient, Takeda, Regeneron, Merz, Iroko, Bioiberica, Crealta/Horizon and Allergan pharmaceuticals, WebMD, UBM LLC and the American College of Rheumatology. JAS serves as the principal investigator for an investigator-initiated study funded by Horizon pharmaceuticals through a grant to DINORA, Inc., a 501 (c)(3) entity. JAS is a member of the executive of OMERACT, an organization that develops outcome measures in rheumatology and receives arms-length funding from 36 companies; a member of the American College of Rheumatology's (ACR) Annual Meeting Planning Committee (AMPC); Chair of the ACR Meet-the-Professor, Workshop and Study Group Subcommittee; and a member of the Veterans Affairs Rheumatology Field Advisory Committee. JAS is the editor and Director of the UAB Cochrane Musculoskeletal Group Satellite Center on Network Meta-analysis. DC has no conflicts to declare. There are no nonfinancial competing interests for any of the authors.

Ethics/IRB Approval and Consent to Participate: The University of Alabama at Birmingham's Institutional Review Board approved this study and waived the need for informed consent for this database study. All investigations were conducted in conformity with ethical principles of research.

\section{REFERENCES}

1. Durward WF. Letter: Gout and hypothyroidism in males. Arthritis Rheum 1976; 19:123.

2. Erickson AR, Enzenauer RJ, Nordstrom DM, Merenich JA. The prevalence of hypothyroidism in gout. Am J Med 1994;97:231-4.

3. Singh JA, Hodges JS, Toscano JP, Asch SM. Quality of care for gout in the US needs improvement. Arthritis Rheum 2007;57:822-9.

4. Marrie RA, Yu BN, Leung S, Elliott L, Warren S, Wolfson C, et al. The incidence and prevalence of thyroid disease do not differ in the multiple sclerosis and general populations: a validation study using administrative data. Neuroepidemiology 2012;39:135-42.

5. Tonelli M, Wiebe N, Fortin M, Guthrie B, Hemmelgarn BR, James MT, et al. Methods for identifying 30 chronic conditions: application to administrative data. BMC Med Inform Decis Mak 2015;15:31.

6. Kvetny J, Heldgaard PE, Bladbjerg EM, Gram J. Subclinical hypothyroidism is associated with a low-grade inflammation, increased triglyceride levels and predicts cardiovascular disease in males below 50 years. Clin Endocrinol (Oxf) 2004;61:232-8. 